\title{
Exogenous normal lymph reduces liver injury induced by lipopolysaccharides in rats
}

\author{
Z.G. Zhao*, L.L. Zhang*, C.Y. Niu and J. Zhang \\ Institute of Microcirculation, Hebei North University, Zhangjiakou, Hebei, China
}

\begin{abstract}
The liver is one of the target organs damaged by septic shock, wherein the spread of endotoxins begins. This study aimed to investigate the effects of exogenous normal lymph (ENL) on lipopolysaccharide (LPS)-induced liver injury in rats. Male Wistar rats were randomly divided into sham, LPS, and LPS + ENL groups. LPS $(15 \mathrm{mg} / \mathrm{kg})$ was administered intravenously via the left jugular vein to the LPS and LPS + ENL groups. At $15 \mathrm{~min}$ after the LPS injection, saline or ENL without cell components $(5 \mathrm{~mL} / \mathrm{kg})$ was administered to the LPS and LPS + ENL groups, respectively, at a rate of $0.5 \mathrm{~mL} / \mathrm{min}$. Hepatocellular injury indices and hepatic histomorphology, as well as levels of P-selectin, intercellular adhesion molecule 1 (ICAM-1), myeloperoxidase (MPO), and $\mathrm{Na}^{+}-\mathrm{K}^{+}$-ATPase, were assessed in hepatic tissues. Liver tissue damage occurred after LPS injection. All levels of alanine aminotransferase (ALT) and aspartate aminotransferase (AST) in plasma as well as the wet/dry weight ratio of hepatic tissue in plasma increased. Similarly, P-selectin, ICAM-1, and MPO levels in hepatic tissues were elevated, whereas $\mathrm{Na}^{+}-\mathrm{K}^{+}$-ATPase activity in hepatocytes decreased. ENL treatment lessened hepatic tissue damage and decreased levels of AST, ALT, ICAM-1, and MPO. Meanwhile, the treatment increased the activity of Na ${ }^{+}-K^{+}-A_{T P a s e}$. These results indicated that ENL could alleviate LPS-induced liver injury, thereby suggesting an alternative therapeutic strategy for the treatment of liver injury accompanied by severe infection or sepsis.
\end{abstract}

Key words: Exogenous normal lymph; Lipopolysaccharide; Liver injury; Intercellular adhesion molecule 1; Myeloperoxidase; $\mathrm{Na}^{+}-\mathrm{K}^{+}$-ATPase

\section{Introduction}

The liver is a target organ that is damaged during the pathogenesis of septic shock. The spread of endotoxins usually begins from this organ, which contributes to the development of multiple-organ dysfunction syndrome; therefore, liver protection is significant in endotoxemia treatment (1-3). Numerous studies have been conducted on liver injury after serious infection or sepsis $(4,5)$, but no effective treatment for liver injury induced by septic shock has been reported. Therefore, the search for effective liver treatment is essential. Previous studies have found that normal lymph has beneficial therapeutic effects on microcirculatory disturbances in rats with hemorrhagic shock; its effect is significantly better than that by the same amount of normal saline and of albumin (6). Exogenous normal lymph (ENL) from healthy dogs has an alleviating effect on microcirculation disturbances and abnormal hemorheological properties in rats with disseminated intravascular coagulation induced by Dextran 500 (7). In addition, ENL could alleviate acute lung injury and acute kidney injury in rats with endotoxic shock induced by lipopolysaccharides (LPS) $(8,9)$. Cheng et al. (10) reported that normal mesenteric lymph could blunt the pulmonary inflammatory response to endotoxin. The effect of ENL on acute liver injury induced by septic shock remains unclear. The present study evaluated the effect of ENL on LPS-induced liver injury and its underlying mechanisms.

\section{Material and Methods}

\section{Animals}

A total of 60 healthy and pathogen-free male Wistar rats (Chinese Academy of Medical Sciences Animal Breeding Center, Beijing, China), weighing 240 to $300 \mathrm{~g}$, were used in this study. The rats were maintained under barrier-sustained conditions with a 12:12-h light/dark cycle at a constant temperature $\left(23 \pm 2^{\circ} \mathrm{C}\right)$, with access to standard laboratory food and water. Before the

Correspondence: C.Y. Niu and/or J. Zhang, Institute of Microcirculation, Hebei North University, Diamond South Road 11, Zhangjiakou 075000, China. Fax: +86-313-402-9168. E-mail: ncylxf@126.com and/or zhangjing55155@163.com

${ }^{*}$ These authors contributed equally to this study.

Received June 5, 2013. Accepted October 11, 2013. First published online January 17, 2014. 
experiment, the rats were fasted for $12 \mathrm{~h}$ but had access to water. Three healthy beagles were used for the preparation of ENL. All animal experiments were conducted in compliance with the guidelines of the Hebei North University Animal Care Committee. All efforts were made to minimize the suffering of the animals.

\section{Preparation of ENL}

After intravenous anesthesia with $25 \mathrm{mg} / \mathrm{kg}$ pentobarbital sodium, a midline laparotomy was performed on healthy beagles, and their normal mesenteric lymph was continuously collected for $30 \mathrm{~min}$ into sterile heparinwetted test tubes, according to our laboratory protocols $(7-9,11)$. Lymph samples were centrifuged at $315 \mathrm{~g}$ for $15 \mathrm{~min}$ to remove all cellular components and stored at $-80^{\circ} \mathrm{C}$ in an ultra-low freezer (Thermo Electron, USA) prior to the experiment.

\section{Experimental protocol}

All rats were anesthetized with $50 \mathrm{mg} / \mathrm{kg} 1 \%$ pentobarbital sodium and were randomly assigned to the sham, LPS, and LPS + ENL groups $(n=20)$. The left jugular vein and the right carotid artery were aseptically separated from the surrounding tissues and cannulated using a microcatheter for drug administration. After the surgical procedure was completed, rats were allowed to adjust for $30 \mathrm{~min}$. LPS (15 mg/kg; Escherichia coli O111:B4; Sigma, USA) was then injected via the left jugular vein using an infusion pump (ZCZ-50; Zhejiang University Medical Ltd., China), within $10 \mathrm{~min}$ after surgery, in the LPS and LPS + ENL groups. LPS was dissolved to a concentration of $10 \mathrm{mg} / \mathrm{mL}$ in normal saline. At $15 \mathrm{~min}$ after LPS injection, ENL was injected via the left jugular vein at a rate of $0.5 \mathrm{~mL} / \mathrm{min}$ for 9 to $12 \mathrm{~min}$ in the LPS +ENL group. The ENL dose was $5 \mathrm{~mL} / \mathrm{kg}$, based on previous experiments $(9,11)$. The LPS group received injections with the same amount of normal saline instead of ENL, and the sham group received normal saline only, without surgery.

\section{Evaluation of liver injury}

Six hours after LPS injection, the hepatic tissue from a designated position was collected from the rats and fixed in $10 \%$ neutral buffered formalin. The samples were dehydrated using a graded ethanol series and were embedded in paraffin, as previously described $(9,11)$. Each paraffin block was processed into $5-\mu \mathrm{m}$ thick slices that were stained with hematoxylin and eosin. Hepatic morphological changes were observed using light microscopy (BH-2, Olympus, Japan), and pictures were taken using a digital camera (4500, Nikon, Japan), according to the standard methods of our laboratory.

A 3-mL blood sample was obtained from the carotid artery 3 or $6 \mathrm{~h}$ after LPS administration $(n=10)$. Plasma was collected by centrifugation at $850 \mathrm{~g}$ for $10 \mathrm{~min}$ and stored at $-80^{\circ} \mathrm{C}$. The activities of alanine aminotransferase
$(\mathrm{ALT})$ and aspartate aminotransferase (AST) in plasma were examined using a commercial kit from Randox Laboratories Ltd. (China) with an automatic biochemical analyzer (Aeroset; Abbott, USA).

Six hours after LPS injection, hepatic tissues were harvested, and their wet weights were immediately measured after blotting with filter paper. The hepatic tissues were embedded in an electric thermostatic drum wind-drying oven (GZX-9070MBE, Boxun Ltd., China) and baked at $80^{\circ} \mathrm{C}$ for $12 \mathrm{~h}$ until the weight remained constant. The dry weight of the hepatic tissue samples was measured and their wet/dry weight ratios (W/D) were calculated.

\section{Preparation of hepatic homogenate}

Hepatic tissue from a designated position was collected from rats at 3 or $6 \mathrm{~h}$ after LPS administration. The tissue samples were homogenized in 1:9 (w/v) normal saline for $30 \mathrm{~s}$ and then centrifuged at $850 \mathrm{~g}$ at $0^{\circ}$ to $4^{\circ} \mathrm{C}$ for $10 \mathrm{~min}$ using a Labofuge 400R supercentrifuge (Thermo Fisher Scientific, USA). The supernatant was stored at $-80^{\circ} \mathrm{C}$ until further use.

\section{Measurement of P-selectin and intercellular adhesion molecule-1 (ICAM-1)}

P-selectin and ICAM-1 contents of the hepatic homogenate were determined using rat enzyme-linked immunosorbent assay kits, according to the manufacturer's instructions. The P-selectin kit was purchased from Yuanye Biotechnology (China), whereas the ICAM-1 kit was purchased from Bosde Biotechnology (China). Protein content of the homogenates was quantified using the Coomassie brilliant blue colorimetric method.

\section{Measurement of myeloperoxidase (MPO)}

MPO activity of the hepatic homogenates was measured using the hydrogen peroxide method (12) with an MPO kit (Jiancheng Biotechnology, China), according to the manufacturer's instructions. A unit of MPO activity represents the amount of enzyme that can reduce $1 \mu \mathrm{mol} /$ min hydrogen peroxide. Results are reported as units of MPO per gram of protein.

\section{Measurement of $\mathrm{Na}^{+}-\mathrm{K}^{+}$-ATPase}

$\mathrm{Na}^{+}-\mathrm{K}^{+}$-ATPase activity in the hepatic homogenates was measured using the phosphorus fixing method (13) with an ATPase kit (Jiancheng Biotechnology), according to the manufacturer's instructions. A unit of ATPase activity represents the amount of enzyme that will produce $1 \mu \mathrm{mol} / \mathrm{h}$ inorganic phosphorus from the hydrolysis of ATP to ADP. Results are reported as units of ATPase per milligram of protein.

\section{Statistical analysis}

Data are reported as means $\pm S D$ and were analyzed using the SPSS (version 16.0, USA) software. 
Comparisons among groups were conducted using oneway ANOVA. When the $F$ value was significant $(P<0.05)$, the Student-Newman-Keuls test was performed to compare differences within each group. Values with $\mathrm{P}<0.05$ were considered to be significant.

\section{Results}

Effect of ENL on hepatic histomorphology in LPS rats

The clear architecture of hepatic lobules, with their regular arrayed hepatic cords, as well as the uniformsized hepatic cells with round nuclei and clear karyolemmas were observed in the control group (Figure 1A). In the LPS group, the central vein and its surrounding hepatic sinusoid were dilated and congested. Furthermore, the hepatic cords were disorganized. Hepatic cells showed turbid swelling, steatosis, and cytoplasmic vacuolation. Neutrophil and accidental eosinophil neutrophil infiltrations were found in the LPS group (Figure 1B). In the LPS + ENL group, the central vein and surrounding hepatic sinusoid had slight dilations and congestion. The hepatic cords were relatively unimpaired, and the architecture of hepatic lobules was normal. Hepatic cells were of uniform size, with clear karyolemmas and slight turbid swelling, and neutrophil infiltrations were occasionally observed (Figure 1C).

\section{Effect of ENL on indices of hepatocellular injury in LPS rats}

Three and six hours after LPS injection, activities of AST and ALT in plasma of the LPS and LPS + ENL groups were significantly higher than those of the sham group $(P<0.05)$. These two indices in the LPS $+E N L$ group at $6 \mathrm{~h}$ were lower than those in the LPS group $(\mathrm{P}<0.05$; Figure 2).
Effect of ENL on W/D of hepatic tissue in LPS rats

The W/D of liver in the LPS $(3.56 \pm 0.22)$ and LPS + ENL (3.52 \pm 0.10$)$ groups was evidently higher than that of the sham group $(3.29 \pm 0.07)$. No significant differences were observed between the LPS and LPS + ENL groups.

\section{Effect of ENL on P-selectin of hepatic tissue in LPS rats}

Three hours after LPS injection, P-selectin content of the hepatic homogenate in the LPS group (20.56 \pm $5.88 \mathrm{ng} / \mathrm{g}$ protein) was significantly higher than that of the sham group $(12.10 \pm 3.40 \mathrm{ng} / \mathrm{g}$ protein). No significant differences were observed between the LPS +ENL group $(15.32 \pm 5.46 \mathrm{ng} / \mathrm{g}$ protein) and the sham or LPS groups.

\section{Effect of ENL on ICAM-1 of hepatic tissue in LPS rats}

Three and six hours after LPS injection, ICAM-1 content of the hepatic homogenate in the LPS group was significantly increased compared with the sham group $(P<0.05)$. However, no significant differences were observed between the sham and LPS + ENL groups $(P>0.05)$. Compared with the LPS group, ICAM-1 content in the LPS +ENL group was lower at $3 \mathrm{~h}(\mathrm{P}<0.05)$, although no significant differences were observed at $6 \mathrm{~h}$ $(\mathrm{P}>0.05$; Figure 3).

\section{Effect of ENL on MPO of hepatic tissue in LPS rats}

MPO activity of the hepatic homogenates in the LPS and LPS + ENL groups at 3 and $6 \mathrm{~h}$ after LPS administration was significantly increased, compared with that of the sham group $(P<0.05$; Figure 4). MPO activity of the LPS + ENL group at $3 \mathrm{~h}$ was significantly lower than that of the LPS group $(\mathrm{P}<0.05)$.
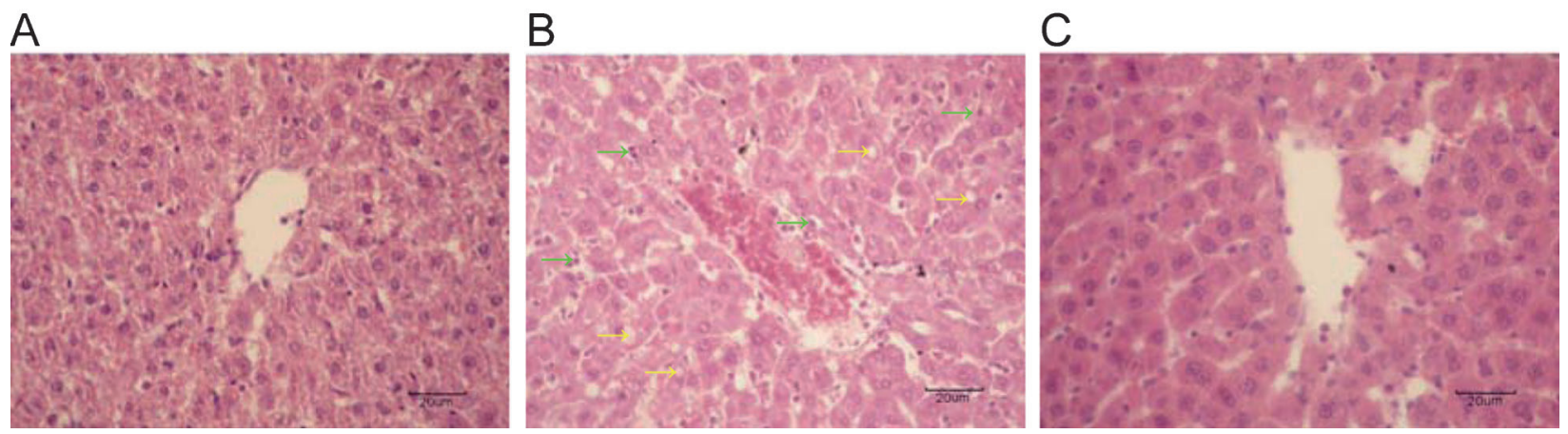

Figure 1. Effect of exogenous normal lymph (ENL) on hepatic morphology in rats (HE staining, $400 \times$, magnification bar: $20 \mu \mathrm{m}$ ). Hepatic tissue was collected $6 \mathrm{~h}$ after lipopolysaccharide (LPS) injection in LPS and LPS +ENL groups, or the corresponding time in the sham group $(\mathrm{n}=3)$. A, Sham group, regular arrayed hepatic cords, as well as the uniform-sized hepatic cells with round nuclei and clear karyolemmas were observed; $B$, LPS group, the central vein and its surrounding hepatic sinusoid were dilated and congested, the hepatic cells showed turbid swelling, steatosis, and cytoplasmic vacuolation (yellow arrows), neutrophil and accidental eosinophil neutrophil infiltrations (green arrows) were found; C, LPS +ENL group, the hepatic cords were relatively unimpaired, and the architecture of hepatic lobules was normal, the changes of hepatic structure were slighter than those of the LPS group. 

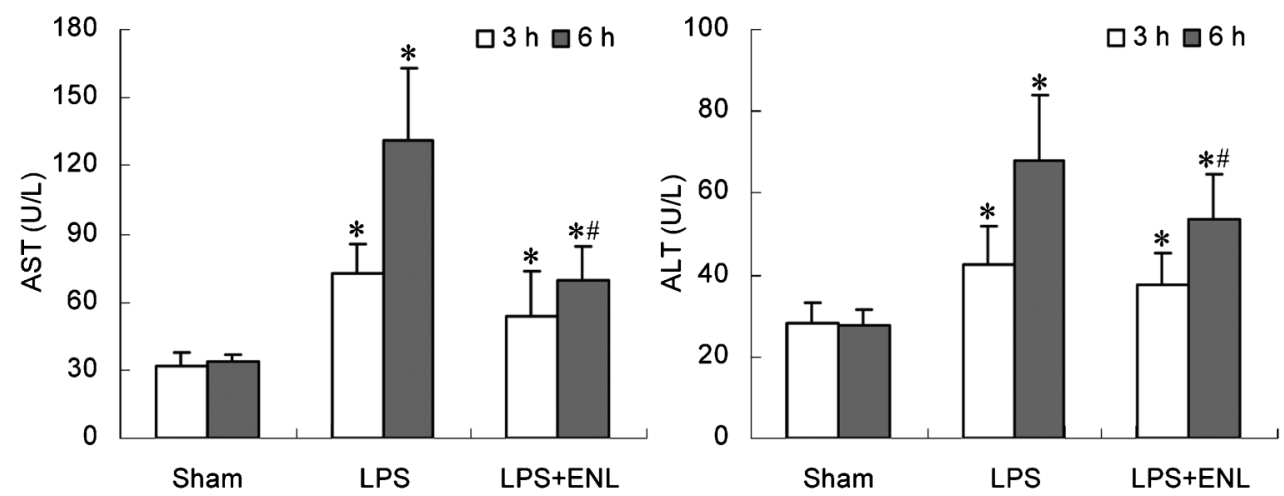

Figure 2. Effect of exogenous normal lymph (ENL) on indices of hepatocellular injury in rats. Data are reported as means $\pm S D(n=10)$. ${ }^{*} \mathrm{P}<0.05$ vs the sham group; ${ }^{\#} \mathrm{P}<0.05$ vs the lipopolysaccharide (LPS) group (one-way ANOVA). AST: aspartate aminotransferase; ALT: alanine aminotransferase.

\section{Effect of ENL on $\mathrm{Na}^{+}-\mathrm{K}^{+}$-ATPase of hepatic tissue in LPS rats}

$\mathrm{Na}^{+}-\mathrm{K}^{+}$-ATPase activity of the hepatic homogenate in the LPS group at 3 and $6 \mathrm{~h}$ after LPS administration, as well as that of the LPS + ENL group at $6 \mathrm{~h}$, were obviously decreased, compared with that of the sham group $(P<0.05$; Figure 5). In contrast, at $3 \mathrm{~h}$ after LPS injection, $\mathrm{Na}^{+}-\mathrm{K}^{+}$-ATPase activity in the LPS +ENL group was significantly higher than that in the LPS group $(P<0.05)$, but not significantly different from that of the sham group $(P>0.05)$.

\section{Discussion}

In this study, we found that administration of ENL could significantly reduce LPS-induced increases in AST and ALT levels in plasma and attenuate the degree of hepatic tissue damage. Liver injury induced by LPS injection was prevented by treatment with ENL. A distinct increase in the W/D ratio of hepatic tissues in the LPS

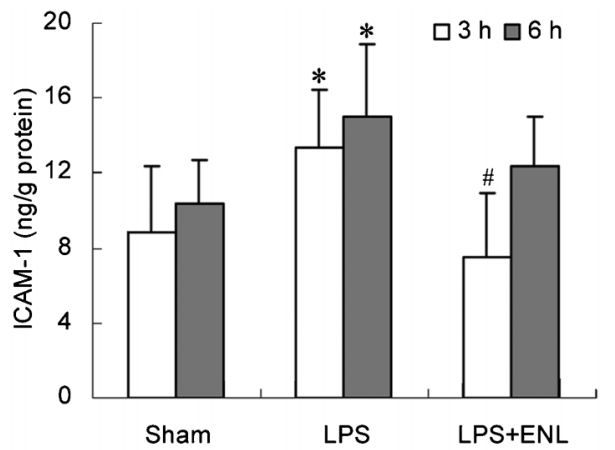

Figure 3. Effect of exogenous normal lymph (ENL) on intercellular adhesion molecule-1 (ICAM-1) of hepatic homogenate in rats. Data are reported as means $\pm S D(n=10)$. ${ }^{*} P<0.05$ vs the sham group; ${ }^{\#} \mathrm{P}<0.05$ vs the lipopolysaccharide (LPS) group (one-way ANOVA) group of rats was observed, thereby indicating that increased moisture in hepatic tissue was induced by LPS by a mechanism related to vascular endothelial cell (VEC) damage induced by LPS (14). Consequently, vascular hyperpermeability occurred. However, the W/D ratio of hepatic tissues was not changed in the LPS + ENL group. This was probably caused by LPS-induced damage to VECs within $15 \mathrm{~min}$. ENL treatment was unable to restore the damaged VECs within $6 \mathrm{~h}$. Therefore, a longer time period after ENL treatment is recommended for further study.

Experimental investigations show that systemic inflammatory response syndrome (SIRS) is an important indication of organ injury (15). LPS is known to induce SIRS in animal models, and the compound has been widely exploited for the study of SIRS-related conditions and for drug development $(16,17)$. Polymorphonuclear neutrophils (PMNs) contribute to LPS-induced SIRS because PMNs are target cells of LPS attack and effector cells of inflammatory mediator release (18). Therefore, we explored the mechanisms by which ENL attenuates liver

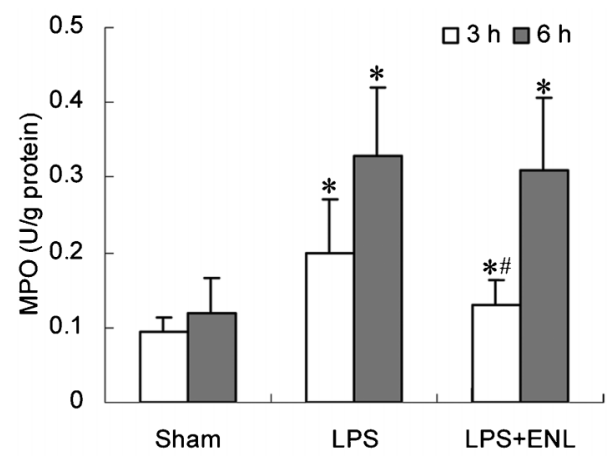

Figure 4. Effect of exogenous normal lymph (ENL) on myeloperoxidase (MPO) activity of hepatic tissue in rats. Data are reported as means $\pm S D(n=10)$. ${ }^{*} P<0.05$ vs the sham group; ${ }^{*} P<0.05$ $v s$ the lipopolysaccharide (LPS) group (one-way ANOVA). 


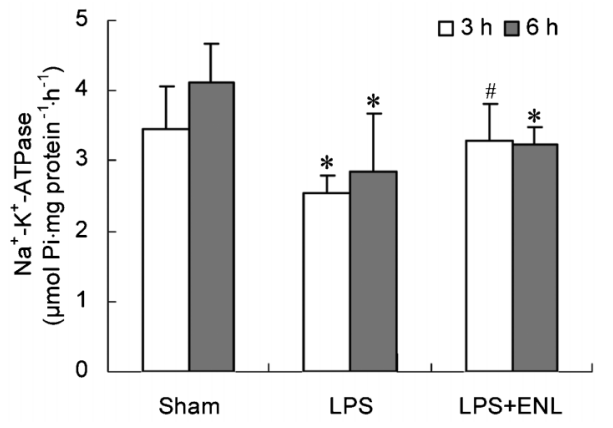

Figure 5. Effect of exogenous normal lymph (ENL) on $\mathrm{Na}^{+}-\mathrm{K}^{+}-$ ATPase activity of hepatic homogenate in rats. Data are reported as means $\pm S D(n=10)$. ${ }^{*} P<0.05$ vs the sham group; ${ }^{\#} P<0.05$ $v s$ the lipopolysaccharide (LPS) group (one-way ANOVA).

injury from PMNs. In general, LPS could activate VECs, and PMNs are generally activated by LPS. An increase in the expression of these cells releases cell adhesion molecules such as P-selectin and ICAM-1, which promote the adhesion, aggregation, and sequestration of PMNs in tissues that may cause SIRS (19). In the current study, the P-selectin level at $3 \mathrm{~h}$ was elevated as the ICAM-1 level and MPO activity at 3 and $6 \mathrm{~h}$ were increased in hepatic tissues of the LPS group. ENL administration reduced ICAM-1 levels and MPO activity of hepatic tissues at $3 \mathrm{~h}$, but not at $6 \mathrm{~h}$. MPO activity in a specific tissue is significantly correlated with the number of PMNs in affected tissues (20). Therefore, LPS induces PMN adhesion via increased P-selectin and ICAM-1 levels. ENL administration is beneficial for reducing liver injury by decreasing the PMN-mediated inflammatory response. In addition, we found that normal lymph might reduce PMNs attached to the venular wall and improve blood flow for microcirculation, as well as decrease the levels of $\mathrm{P}$ selectin and ICAM-1 in plasma (21). This result is an important supplement to the findings of the current study.

ATPase is a membrane-bound protein found in all cells, and it maintains a chemical and electrical gradient across the cell membrane $(22,23)$. The degree of cellular injury is closely related to the activity of ATPase. Any factors that may alter ATPase activity of the cytomembrane will suppress the transportation of ions into and from these cells (24). A change in the electrochemical gradient may lead to a wide array of cellular errors that ultimately aggravate the dysfunction and morphological damage of organs (25). ATPase in membranes includes $\mathrm{Na}^{+}-\mathrm{K}^{+}$-ATPase, $\mathrm{Na}^{+}-\mathrm{H}^{+}$-ATPase, $\mathrm{Ca}^{2+}$-ATPase, $\mathrm{Ca}^{2+}-\mathrm{Mg}^{2+}-\mathrm{ATP}$ ase, etc. Among them, $\mathrm{Na}^{+}-\mathrm{K}^{+}-$ ATPase is the most important, and it is involved in the formation of cell edema. Therefore, we examined $\mathrm{Na}^{+}$$\mathrm{K}^{+}$-ATPase activity as an indicator of membrane pump function of hepatocytes. The results of this study showed that $\mathrm{Na}^{+}-\mathrm{K}^{+}$-ATPase activity in hepatic tissues was obviously decreased at 3 and $6 \mathrm{~h}$ after LPS injection, and a low dose of ENL improved $\mathrm{Na}^{+}-\mathrm{K}^{+}$-ATPase activity. The present data implied that improvement of membrane pump activity is an important mechanism of ENL in alleviating acute liver injury induced by LPS.

In addition, a previous study in our laboratory (26) showed that ENL administration could increase hepatic microregional blood perfusion at 270-360 min after LPS injection, which might be one of the mechanisms of ENL alleviating LPS-induced liver injury. Meanwhile, the results also showed that ENL administration markedly prolonged survival time of the endotoxically shocked rats from $7.21 \pm 1.33$ to $11.80 \pm 2.67 \mathrm{~h}$, which further confirmed the beneficial effect of ENL intervention on endotoxic shock.

Several limitations were observed in the present study. First, the model of LPS-induced liver injury does not completely reflect all types of acute liver injury in the human body. Second, although LPS injections were slowly administered for $10 \mathrm{~min}$ in the animal model to imitate sepsis in clinical practice, several differences between animal models and human patients were apparent. Third, the long-term effects of ENL treatment of LPS-induced liver injury have to be clarified. Nonetheless, the current results are clinically significant for the treatment of septic shock. Finally, ENL administration was performed in this study at 15 min after LPS injection. However, whether ENL administration after $15 \mathrm{~min}$ has a beneficial effect is still not clear, which is one limitation of the current study, and the effect of ENL administration on endotoxic shock at multiple time points after LPS injection should be investigated in the future.

The amount of lymph used in this experiment was only one-fifteenth of whole blood volume, and an equal volume of normal saline was administered to the LPS group. Consequently, the interventions of normal lymph are difficult to explain by supplementary blood volume and hemodilution. Treatment with a low dose of normal lymph may lessen reperfusion injury induced by fluid resuscitation, thereby allowing for a wider range of prospective clinical applications. It should be pointed out that, although the composition of normal lymph is similar to normal plasma, there are differences between them, such as hypoviscosity, hypoproteins, hyperlipoproteins, and so on $(27,28)$, which were involved in the normal lymph ameliorating microcirculation or energy metabolism and, as a result, alleviating organ injury. In addition, Interewicz et al. (29) reported that unknown proteins of low molecular weight were found in lymph but not plasma, using the ProteinChip SELDI MS system. We believe that these unknown proteins may partly explain the observed beneficial effect of normal lymph, alleviating liver injury. However, further research is needed. Meanwhile, ENL obtained from healthy dogs had no immunological rejection in our previous studies $(7-9,21)$ or in the current study. Therefore, healthy dogs may be safely used as donors of ENL. Normal lymph can also be obtained from 
large animals such as pigs and sheep. This study provided experimental evidence of lymph development and a novel target for the treatment of septic shock.

We conclude that ENL can alleviate LPS-induced liver injury. The mechanism involved is related to a decrease in neutrophil adhesions and improvement of $\mathrm{Na}^{+}-\mathrm{K}^{+}$ATPase activity. These data present an alternative therapeutic strategy for the treatment of liver injury accompanied by severe infection or sepsis.

\section{References}

1. Korish AA, Arafa MM. Propolis derivatives inhibit the systemic inflammatory response and protect hepatic and neuronal cells in acute septic shock. Braz J Infect Dis 2011; 15: 332-338.

2. Hozumi H, Tada R, Murakami T, Adachi $\mathrm{Y}$, Ohno N. Comparative analysis of hepatic CD14 expression between two different endotoxin shock model mice: relation between hepatic injury and CD14 expression. PLoS One 2013; 8: e53692, doi: 10.1371/journal.pone.0053692.

3. Marzocco S, Di Paola R, Ribecco MT, Sorrentino R, Domenico B, Genesio M, et al. Effect of methylguanidine in a model of septic shock induced by LPS. Free Radic Res 2004; 38: 1143-1153, doi: 10.1080/107157604100017 25517.

4. Szabo C, Modis K. Pathophysiological roles of peroxynitrite in circulatory shock. Shock 2010; 34 (Suppl 1): 4-14, doi: 10.1097/SHK.0b013e3181e7e9ba.

5. Gonzalez-Teran B, Cortes JR, Manieri E, Matesanz N, Verdugo A, Rodriguez ME, et al. Eukaryotic elongation factor 2 controls TNF-alpha translation in LPS-induced hepatitis. J Clin Invest 2013; 123: 164-178, doi: 10.1172/ JCl65124.

6. Niu CY, Zhang J, Yao T, Fan G. The antagonistic effect of lymph on shock in rat. Lymphology 1998; 31 (Suppl): 159162.

7. Niu CY, Zhao ZG, Zhang YP, Hou YL, Li JJ, Jiang H, et al. Exogenous normal lymph alleviates microcirculation disturbances and abnormal hemorheological properties in rats with disseminated intravascular coagulation. Braz J Med Biol Res 2013; 46: 138-147, doi: 10.1590/1414-431X2012 2378.

8. Fan G, Zhao ZG, Niu CY, Yang YQ, Zhang J. [Effects of normal lymph on myeloperoxidase activity and adenosine triphosphatase in rats with endotoxic shock]. Zhongguo Wei Zhong Bing Ji Jiu Yi Xue 2006; 18: 721-723.

9. Zhao ZG, Niu CY, Zhang LL, Zhang J, Han R, Zhang YP, et al. Exogenous normal lymph alleviates lipopolysaccharide-induced acute kidney injury in rats. Ren Fail 2013; 35: 806-811, doi: 10.3109/0886022X.2013.794680.

10. Cheng AM, Moore EE, Masuno T, Escobar GA, Sarin EL, Johnson JL, et al. Normal mesenteric lymph blunts the pulmonary inflammatory response to endotoxin. J Surg Res 2006; 136: 166-171, doi: 10.1016/j.jss.2006.05.013.

11. Zhao ZG, Niu CY, Zhang YP, Hou YL, Li JJ, Jiang $H$. Exogenous normal lymph alleviating kidney injury by improving coagulation function in disseminated intravascular coagulation rats. Ren Fail 2012; 34: 221-226.

12. Anderson BO, Brown JM, Shanley PF, Bensard DD, Harken

\section{Acknowledgments}

Research supported by scientific research projects of the Education Department in Hebei Province (\#2005311, \#2007407) and the Foundation of Hundred Innovative Talents in Universities of Hebei Province, China (\#CPRC047, \#CPRCII026).

$\mathrm{AH}$. Marginating neutrophils are reversibly adherent to normal lung endothelium. Surgery 1991; 109: 51-61.

13. Fedorova OV, Lakatta EG, Bagrov AY. Endogenous $\mathrm{Na}, \mathrm{K}$ pump ligands are differentially regulated during acute $\mathrm{NaCl}$ loading of Dahl rats. Circulation 2000; 102: 3009-3014, doi: 10.1161/01.CIR.102.24.3009.

14. Dayoub JC, Ortiz F, Lopez LC, Venegas C, Del PinoZumaquero A, Roda O, et al. Synergism between melatonin and atorvastatin against endothelial cell damage induced by lipopolysaccharide. J Pineal Res 2011; 51: 324-330, doi: 10.1111/j.1600-079X.2011.00892.x.

15. Zhang $Q$, Raoof $M$, Chen $Y$, Sumi $Y$, Sursal $T$, Junger $W$, et al. Circulating mitochondrial DAMPs cause inflammatory responses to injury. Nature 2010; 464: 104-107, doi: 10 1038/nature08780.

16. Remick DG, Newcomb DE, Bolgos GL, Call DR. Comparison of the mortality and inflammatory response of two models of sepsis: lipopolysaccharide vs cecal ligation and puncture. Shock 2000; 13: 110-116, doi: 10.1097/00024 382-200013020-00004.

17. Hollenberg SM, Broussard M, Osman J, Parrillo JE. Increased microvascular reactivity and improved mortality in septic mice lacking inducible nitric oxide synthase. Circ Res 2000; 86: 774-778, doi: 10.1161/01.RES.86.7.774.

18. Nakamori $\mathrm{Y}$, Koh T, Ogura H, Tanaka H, Fujimi S, Kasai K, et al. Enhanced expression of intranuclear NF-kappa B in primed polymorphonuclear leukocytes in systemic inflammatory response syndrome patients. J Trauma 2003; 54: 253-260, doi: 10.1097/01.TA.0000037096.73714.E6.

19. Power C, Wang JH, Sookhai S, Wu QD, Redmond HP. Proinflammatory effects of bacterial lipoprotein on human neutrophil activation status, function and cytotoxic potential in vitro. Shock 2001; 15: 461-466, doi: 10.1097/00024382200115060-00009.

20. Reino DC, Pisarenko V, Palange D, Doucet D, Bonitz RP, Lu $Q$, et al. Trauma hemorrhagic shock-induced lung injury involves a gut-lymph-induced TLR4 pathway in mice. PLOS One 2011; 6: e14829, doi: 10.1371/journal.pone.0014829.

21. Zhang LL, Zhao ZG, Niu CY, Zhang J. [Interference effects of lymph plasma on endotoxic shock in rats]. Zhongguo Ying Yong Sheng Li Xue Za Zhi 2011; 27: 75-80.

22. Murphy E, Eisner DA. Regulation of intracellular and mitochondrial sodium in health and disease. Circ Res 2009; 104: 292-303, doi: 10.1161/CIRCRESAHA.108.189050.

23. Billimoria FR, Dave BN, Katyare SS. Neonatal hypothyroidism alters the kinetic properties of $\mathrm{Na}^{+}, \mathrm{K}^{+}$-ATPase in synaptic plasma membranes from rat brain. Brain Res Bull 2006; 70: 55-61, doi: 10.1016/j.brainresbull.2006.03.005. 
24. Garces EO, Victorino JA, Veronese FV. [Anticoagulation in continuous renal replacement therapies (CRRT)]. Rev Assoc Med Bras 2007; 53: 451-455, doi: 10.1590/S010442302007000500023.

25. Berger G, Guetta J, Klorin G, Badarneh R, Braun E, Brod V, et al. Sepsis impairs alveolar epithelial function by downregulating Na-K-ATPase pump. Am J Physiol Lung Cell Mol Physiol 2011; 301: L23-L30, doi: 10.1152/ajplung.00010.2010.

26. Zhang LL, Zhang J, Zhao ZG, Zhang YP, Niu CY. [Intervention role of lymph plasma on renal and hepatic blood perfusion in rats with endotoxic shock]. Zhongguo Wei Zhong Bing Ji Jiu Yi Xue 2010; 22: 740-743.
27. Zhao ZG, Niu CY, Hdu YL. [Comparation of rheology and ingredient between normal lymph and blood]. Zhongguo Ying Yong Sheng Li Xue Za Zhi 2009; 25: 482-484.

28. Nanjee MN, Cooke CJ, Wong JS, Hamilton RL, Olszewski WL, Miller NE. Composition and ultrastructure of size subclasses of normal human peripheral lymph lipoproteins: quantification of cholesterol uptake by HDL in tissue fluids. J Lipid Res 2001; 42: 639-648.

29. Interewicz B, Olszewski WL, Leak LV, Petricoin EF, Liotta LA. Profiling of normal human leg lymph proteins using the 2-D electrophoresis and SELDI-TOF mass spectrophotometry approach. Lymphology 2004; 37: 65-72. 